\title{
A longitudinal analysis of maternal depressive symptoms and children's food consumption and weight outcomes
}

\author{
Taryn W Morrissey ${ }^{1, *}$ and Rada K Dagher ${ }^{2}$ \\ 'Department of Public Administration and Policy, School of Public Affairs, American University, 4400 \\ Massachusetts Ave. NW, Washington, DC 20016, USA: ${ }^{2}$ Department of Health Services Administration, School \\ of Public Health, University of Maryland, College Park, MD, USA
}

Submitted 30 July 2013: Final revision received 28 October 2013: Accepted 8 November 2013: First published online 6 January 2014

\begin{abstract}
Objective: Maternal depressive symptoms negatively impact mothers' parenting practices and children's development, but the evidence linking these symptoms to children's obesity is mixed.

Design: We use a large sample to examine contemporaneous and lagged associations between maternal depressive symptoms and children's BMI, obesity and food consumption, controlling for background characteristics.

Setting: Data from the Early Childhood Longitudinal Study-Birth Cohort (ECLS-B), a longitudinal study of children from infancy through kindergarten in the USA, were collected at four waves from 2001 to 2007, when children were 9 months, 2 years, 4 years and $5 \frac{1}{2}$ years of age, through surveys, child assessments and observations. Subjects: A sub-sample of children from the ECLS-B is used ( $n$ 6500).

Results: Between $17 \%$ and 19\% of mothers reported experiencing depressive symptoms; $17 \%$ to $20 \%$ of children were obese. Maternal depressive symptoms were associated with a small decrease in the likelihood her child was obese $(0 \cdot 8$ percentage points) and with lower consumption of healthy foods. The duration of maternal depressive symptoms was associated with higher BMI (0.02 SD) among children whose parents lacked college degrees.

Conclusions: Results indicate that mothers' depressive symptoms have small associations with children's food consumption and obesity. Among children whose parents lack college degrees, persistent maternal depressive symptoms are associated with slightly higher child BMI. Findings highlight the need to control for depression in analyses of children's weight. Interventions that consider maternal depression early may be useful in promoting healthy weight outcomes and eating habits among children.
\end{abstract}

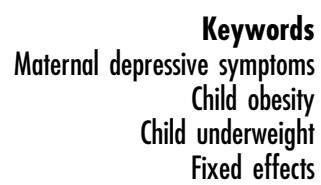

In 2009-2010, $12 \%$ of 2- to 5-year-olds in the USA were considered obese (a BMI at or above the 95th percentile for age and sex in the Centers for Disease Control and Prevention growth charts $)^{(1,2)}$. Childhood obesity is associated with negative health outcomes ${ }^{(3)}$ and an increased likelihood of becoming an obese adult ${ }^{(4)}$. Children in lower socio-economic households are more at risk for overweight and obesity ${ }^{(5)}$. Given its consequences, it is vital to understand the determinants of childhood obesity and to develop strategies to promote healthy weight, particularly during the developmentally important early childhood period ${ }^{(6)}$.

As the primary caregiver in most households ${ }^{(7)}$, mothers' behaviours and interactions with children have important implications for children's development ${ }^{(8)}$. Maternal depressive symptoms disrupt normal maternal-child interactions $^{(9)}$, parenting ${ }^{(10)}$, health behaviours and disease prevention activities ${ }^{(11)}$, and potentially children's weight outcomes. Depression affects an estimated $13-24 \%$ of mothers with young children ${ }^{(12)}$. The first year after birth represents a particularly vulnerable time for maternal depressive symptoms, with research identifying prevalence rates varying from $4 \%$ to $37 \%$ in the $\mathrm{USA}^{(13)}$. The effects of maternal depressive symptoms may be particularly important during the early childhood period, as this is the time when children form attachments with their parents and establish many routines maintained into adulthood ${ }^{(8)}$.

Mothers' depressive symptoms may affect parenting behaviours related to children's eating habits or physical activity in ways that may contribute to obesity among their children $^{(14)}$. For example, some studies show that mothers exhibiting depressive symptoms have a lower likelihood of breast-feeding ${ }^{(15)}$. The exclusive use of breast-feeding until 6 months, with continuation as complementary foods are introduced through 1 year of age, is associated with improved nutrition among infants and a $15-30 \%$ reduction in obesity rates later in life ${ }^{(16)}$. The substitution of 
breast-feeding with bottled formula or cow's milk has been associated with poorer self-regulation and excessive weight gain in infancy, and may lead to an increased likelihood of child overweight at later ages ${ }^{(16)}$. Further, mothers with depression are more likely to be overweight themselves $^{(17)}$ and may model poorer health habits. Establishing and maintaining regular mealtimes, meal preparation or naptime routines is often difficult for families with mothers facing depressive symptoms ${ }^{(10,15)}$, which may lead to skipped or irregular meals and, subsequently, reduced or higher energy intake among their children. Further, children may experience metabolic abnormalities stemming from the increased stress of living with a mother exhibiting depressive symptoms ${ }^{(18)}$.

Indeed, a growing body of evidence suggests that exposure to maternal depressive symptoms may negatively impact young children's growth and health ${ }^{(19,20)}$. However, while some research has linked maternal depressive symptoms to an increased likelihood of being underweight among young children in certain developing countries or ethnic groups in the USA ${ }^{(21,22)}$, other research in Europe, Africa, and North and South America has found no associations $^{(19,23)}$. Similarly, the limited research on maternal depressive symptoms and child height and weight has produced mixed results, with some studies finding positive associations between exposure to maternal depressive symptoms during infancy and their children's subsequent probability of being overweight ${ }^{(14,24)}$ and others finding no association with weight ${ }^{(25,26)}$ or height outcomes ${ }^{(27)}$.

Much of this previous work relies on international or cross-sectional samples. Two notable exceptions using longitudinal data examined children's exposure to maternal depressive symptoms during infancy and their later weight and growth outcomes. One study found that children exposed to maternal depressive symptoms at all three measured time points during infancy were $2 \cdot 1$ times more likely to be overweight in elementary school than their peers ${ }^{(28)}$; however, their sample of 1090 children was relatively advantaged, limiting its generalizability. The second study, using data on 6550 singleton children from the nationally representative Early Childhood Longitudinal Study-Birth Cohort (ECLS-B), found that children exposed to postpartum maternal depressive symptoms had a $40 \%$ increased odds of being in the 10th percentile or lower for measures of height-for-age for children aged 4 and 5 years ${ }^{(29)}$. Although these studies controlled for numerous household, maternal and child characteristics, analyses remain vulnerable to omitted variable bias resulting from factors related to both children's weight and maternal depressive symptoms, such as residential mobility or neighbourhood characteristics (e.g. the availability of parks or healthy foods) $)^{(30,31)}$. Further, both of these studies examined postpartum depression and children's later growth; thus, the contemporaneous associations between mothers' depressive symptoms and children's eating habits and growth remain unclear.
Some research suggests that parenting quality may partially mediate associations between maternal depression and elementary-school children's BMI ${ }^{(14)}$. One study of elementary-school children found links between maternal depressive symptoms and children's BMI, with some evidence that unhealthy eating habits partially mediated this association among boys ${ }^{(32)}$, but research on the dietary mechanisms during the developmentally important early childhood period remain unexplored.

Furthermore, the influences of mothers' depressive symptoms on children's weight outcomes may vary with socio-economic characteristics. Parents' educational level, particularly mothers', is consistently positively associated with children's health and development ${ }^{(8)}$. The weight outcomes of children living in less-educated families may be particularly vulnerable to the effects of mothers' depressive symptoms, as these families may understate the importance of nutrition and regular mealtimes, leading to excessive weight gain ${ }^{(33)}$. Additionally, less-educated families may purchase lower-cost, energy-dense but nutritionally poor foods as opposed to higher-cost, more nutritious foods that tend to be more expensive or more time-consuming to prepare $^{(34)}$. However, to date, while studies control for parent education, as it is negatively related to child overweight $^{(4)}$, the moderating effects of parental education in the relationship between maternal depression and child weight outcomes have not been explored.

The present study investigates the relationship between mothers' depressive symptoms and children's BMI and obesity and whether children's food consumption mediates the expected positive association between maternal depressive symptoms and children's weight. In addition, we examine whether the (expected) association between maternal depressive symptoms and children's obesity is stronger among families with less-educated parents. We use longitudinal, nationally representative data and employ rigorous longitudinal techniques to limit potential biases from unmeasured child and family characteristics.

\section{Methods}

\section{Sample}

We use data from the first four waves of the ECLS-B, a comprehensive, longitudinal study conducted by the National Center for Education Statistics. The baseline sample of approximately 10700 children was designed to be nationally representative of children born in the USA in 2001 with an over-sample of Asian and AmericanIndian children, twins, and low- and very-low-birthweight children.* Data collection for the ECLS-B occurred when the children were 9 months of age, 2 years of age, approximately 4 years of age (the pre-school wave) and

\footnotetext{
* The reported sample sizes are rounded to the nearest fifty, per National Center for Education Statistics regulations regarding disclosure of restricted-use data.
} 
at kindergarten entry ( $5 \frac{1}{2}$ years of age).. Data were collected using parent surveys, direct child assessments and observations of the home and child-care environments at each wave. Our analysis sample includes children who had data on predictor and outcome variables for at least one wave and who were not low or very low birth weight $\uparrow$ (4850-6500 at each wave). The analysis sample differed from those dropped due to missing data or low birth weight. Retained children averaged higher BMI, higher maternal depressive scores and higher maternal pre-pregnancy weight, were more likely to be male and to live with married and more educated parents, but less likely to be a twin or Black, or to live in households under the poverty line. This research received exemption from the American University and University of Maryland Institutional Review Boards.

\section{Measures}

Measures of children's obesity and food consumption serve as outcome variables. At each wave of data collection, measures of children's weight and length/height were collected in the lab. Children removed their shoes, and trained data collectors measured weight and length/height twice. Measures that were more than 5\% apart were conducted a third time. The two closest measurements were then averaged together. At the 9-month wave and for children younger than 24 months at the 2-year wave, a measure of weight-for-length was calculated. At the pre-school and kindergarten entry waves and for children 24 months or older at the 2-year wave, BMI was calculated. For measures collected under 24 months, weight-forlength $Z$-scores, standardized across age and sex, were used as dependent variables. For measures collected at 24 months and older, standardized BMI $Z$-scores were used. A binary measure of obesity (at or above the 95th percentile) by age and sex was calculated at each age using the Centers for Disease Control and Prevention standards ${ }^{(2)}$.

Children's food consumption was reported by parents (usually mothers) using a subset of questions from the Food Consumption Questionnaire (FCQ) at the 4-year and 51/2-year waves. Parents reported the frequency that their children consumed specific foods in the past $7 \mathrm{~d}$. Following previous research using this measure ${ }^{(35)}$, qualitative response categories were translated into continuous measures representing the number of times in the previous $7 \mathrm{~d}$ that the child ate or drank a certain food. Midpoints were used for responses spanning several times (e.g. ' $1-3$ times during the past $7 \mathrm{~d}$ ' was coded as '2 times/week').

\footnotetext{
* In the autumn of 2006, information was collected from all participating children, approximately $75 \%$ of whom were in kindergarten or higher. In the autumn of 2007 , data were collected from the remaining $25 \%$ of participants who had not yet entered kindergarten, as well as from those who were repeating kindergarten in the 2007-08 school year. Only data from the first kindergarten entry wave (2006-07) are used in the present study.

$\uparrow$ Two thousand eight hundred children were considered as having low or very low birth weight and were excluded from our sample.
}

Two indices were created at each wave: (i) a Healthy Food Consumption Index, representing the frequency of consumption of healthy foods (vegetables, fruit, milk and $100 \%$ juice); and (ii) an Unhealthy Food Consumption Index, representing the frequency of consumption of less healthy foods (sugar-sweetened beverages, salty snacks, sweets and fast food). At each wave, Cronbach's $\alpha$ for the Unhealthy Index ranged from 0.53 to 0.57 , and from 0.46 to 0.66 for the Healthy Index. Most questions came from the Centers for Disease Control and Prevention's Youth Risk Behavior Surveillance Survey and have been validated $^{(36)}$. The measure is limited in that it did not collect serving size. See online supplementary material for a table describing the food consumption indices.

Our independent variable of maternal depressive symptoms was measured using a modified version of the Center for Epidemiologic Studies Depression Scale (CES-D: $\alpha=0.87$ to 0.90 at each wave) ${ }^{(37)}$ at 9 months, pre-school and kindergarten entry, and the Depression Scale of the Composite International Diagnostic Interview Short Form $(\mathrm{CIDI}-\mathrm{SF})^{(38)}$ at 2 years. We considered those with a raw score of 9 or higher as experiencing moderate or severe depressive symptoms (a cut-off of 15 or higher is considered severe $)^{(37)}$. The CIDI-SF was originally created for the National Health Interview Survey using questions from the full CIDI. Only the depression questions were included in the ECLS-B $(\alpha=0 \cdot 67)$. If respondents deny symptoms of dysphoria or the persistence of symptoms to the first three 'stem' questions, they are considered as not having a major depressive disorder; if an individual responds affirmatively to at least one stem question, she is asked seven additional branch questions. Mothers who reported an affirmative answer to one of the stem questions were considered as having moderate or severe depressive symptoms. Standardized depression $Z$-scores $($ mean $=0, \mathrm{SD}=1$ ) were generated at each wave and used as predictors in models including all waves of data. Measures of the duration of moderate or severe maternal depressive symptoms at each wave were calculated by summing the number of periods mothers were considered depressed (having moderate/severe depressive symptoms) up to that wave.

Measures of child age in months, gender, race/ethnicity, health insurance coverage, mothers' pre-pregnancy weight, maternal and paternal employment hours (not employed, employed 1-35h/week, employed $35 \mathrm{~h}$ or more/week), family structure (married, not married), poverty status (less than or equal to $100 \%$ of the Federal Poverty Level (FPL), $130 \% \mathrm{FPL}, 185 \% \mathrm{FPL}$ ), whether the family lived in an urban or rural area and data collection wave were included as covariates in the regression models. Parental education, included as a control and moderator, is coded from respondents' reports of mothers' and fathers' education at wave 1 (neither parent graduated high school, at least one parent has a high school degree, at least one parent graduated from college). 


\section{Analyses}

A series of ordinary least squares (OLS) and linear probability models (LPM) were used to predict children's standardized BMI and obesity. First, data were pooled across waves to test cross-sectional associations between measures of maternal depressive symptoms (using continuous scores, binary categories and duration) and children's weight outcomes. Second, within-child fixed-effects (FE) models were used to limit potential biases resulting from confounding factors related to a child's propensity to have a depressed mother and to be obese. FE models use within-child comparisons to predict changes in the outcome (child BMI or obesity status) from changes in the predictor (measures of maternal depressive symptoms). As a result, all time-invariant (fixed) measured and unmeasured confounding factors are controlled, providing more conservative estimates. Third, analogous models as described above were tested, replacing the dependent variable with children's healthy and unhealthy food consumption. Finally, we explore the moderating influences of parental education on the association between maternal depressive symptoms and children's weight outcomes by adding an interaction between maternal depressive symptoms and whether at least one parent graduated from college. Descriptive statistics use sample weights, but because we pool data across four waves, appropriate weights are not available for the pooled sample descriptives or regression estimates. Logistic regression analyses were also conducted; results showed similar patterns and thus we only present the LPM results. Results are available upon request. All statistical analyses were conducted using the statistical software package Stata version 12.

\section{Results}

Table 1 provides weighted descriptive statistics of the analysis sample. At each data wave, between $17 \%$ and $19 \%$ of mothers were considered as having moderate or severe depressive symptoms (5-8\% reported severe symptoms). The prevalence of obesity among children ranged from $17 \%$ to $20 \%$. On average, parents reported their children consumed healthy foods 42-44 times/week and less healthy foods less often (17 times/week).

Results from the LPM and within-child FE models predicting child weight outcomes from maternal depressive symptoms, controlling for background characteristics, are shown in Table 2. Results suggest that the categorical measure of maternal depressive symptoms is associated with a 0.07 decrease in BMI Z-score and a 1.9 percentage point decrease in the likelihood her child is obese. A 1-point (or $1 \mathrm{SD}$ ) increase in mothers' depressive scores is also associated with a 0.8 percentage point decrease in the likelihood of child obesity. By contrast, the FE models found a marginally statistically significant association between the duration of time a mother was considered as having moderate or severe depressive symptoms and a 0.06 increase in her child's BMI Z-score. Like the LPM findings, FE model results also indicate that a 1-point increase in maternal depressive scores is associated with a $0 \cdot 8$ percentage point decrease in the likelihood her child is obese.

To test the moderating effects of parental educational attainment, FE models including interactions between measures of maternal depressive symptoms and parental education were conducted. Results indicate that the duration of moderate or severe maternal depressive symptoms predicts a 0.018 increase in a child's BMI Z-score among families in which neither parent has a bachelor's degree (at wave 1), but not among families in which at least one parent has a bachelor's degree (interaction: $\beta=-0 \cdot 083$, $\mathrm{SE}=0 \cdot 042, P=0 \cdot 047)$.

Table 3 displays results from OLS and FE models predicting children's consumption of healthy and unhealthy foods from measures of mothers' depressive symptoms, controlling for child and family characteristics. Consistent with expectations, maternal depressive symptoms and the duration of moderate or severe maternal depressive symptoms are associated with a lower frequency of healthy food consumption and a greater frequency of unhealthy food consumption in the OLS models. FE models reveal fewer significant associations between changes in maternal depressive symptoms and changes in children's unhealthy food consumption; however, consistent with the OLS results, an increase in measures of maternal depressive symptoms is associated with small decreases in the frequency of consumption of healthy foods.

Although the signs on the coefficients were in the expected direction and the $P$ values approached statistical significance, measures of children's food consumption did not predict children's weight outcomes (results not shown; available upon request); thus, full mediation models including measures of both maternal depressive symptoms and children's food consumption were not conducted.

Several sensitivity analyses were conducted (not shown). First, all main effects models were re-run to exclude wave 2 measures because a different maternal depressive symptoms assessment was used at this wave. Second, all main effects models were re-run to exclude observations for which children were younger than 24 months, when infants' weight-for-length were used instead of weight-for-height measures as dependent variables. For both sets of these models, the patterns of results are largely the same as those described above. Third, LPM and FE models predicting children's weight outcomes from earlier measures of maternal depressive symptoms were tested, finding no lagged associations. Fourth, LPM, OLS and FE models predicting children's weight and food consumption outcomes at kindergarten entry (wave 4) from measures of maternal depressive symptoms at earlier waves across early childhood found no significant 
Table 1 Weighted sample descriptive statistics over the first four waves of data; Early Childhood Longitudinal Study-Birth Cohort, USA, 2001-2007

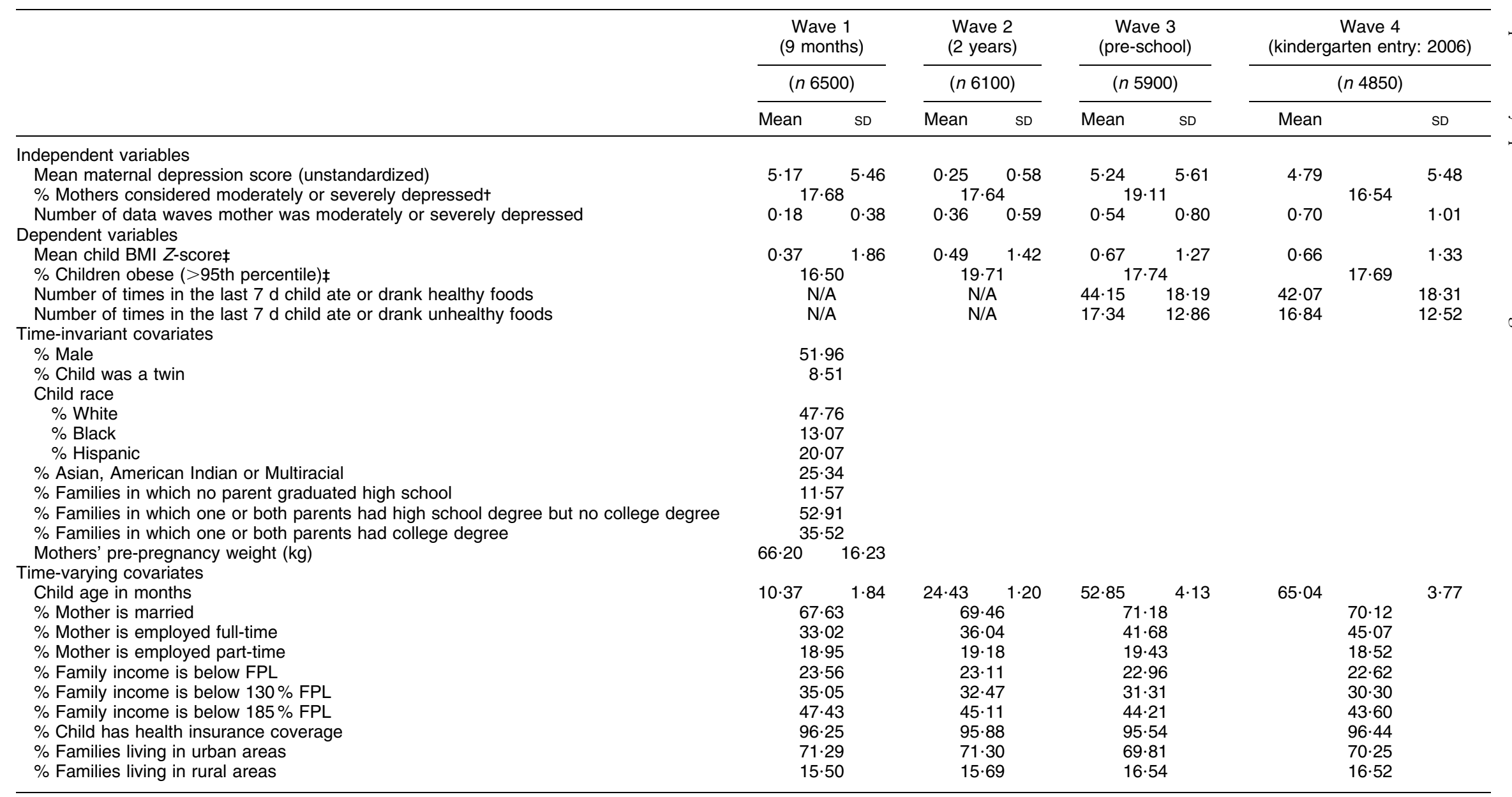

FPL, federal poverty level; N/A, not applicable; NCES, National Center for Education Statistics; CIDI-SF, Composite International Diagnostic Interview Short Form; CES-D, Center for Epidemiologic Studies Depression Scale; CDC, Centers for Disease Control and Prevention.

Data are presented as mean and standard deviation for continuous variables, or as percentages for categorical variables. Sample sizes were rounded to the nearest fifty, in accordance with NCES regulations.

tAt the 2-year wave, mothers reporting $\geq 1$ affirmative answer to the three stem questions on the CIDI-SF were considered moderately or severely depressed; $\geq 1$ affirmative answer to a stem question and $\geq 3$ symptoms to the branch questions were considered severely depressed. At all other waves, mothers scoring $\geq 9$ on the CES-D were considered moderately or severely depressed; mothers scoring $\geq 15$ were considered severely depressed.

fObesity status was calculated using the percentiles for length-for-weight for measures taken before 24 months and weight-for-height (i.e. BMI) at 24 months and older, according to CDC guidelines (at or above the 95 th percentile for age and sex). 
Table 2 Predicting children's BMI Z-scores and obesity (BMI at or above the 95th percentile for age and sex) from measures of maternal depression: ordinary least squares (OLS), linear probability models (LPM) and within-child fixed-effects (FE) models; Early Childhood Longitudinal Study-Birth Cohort, USA, 2001-2007

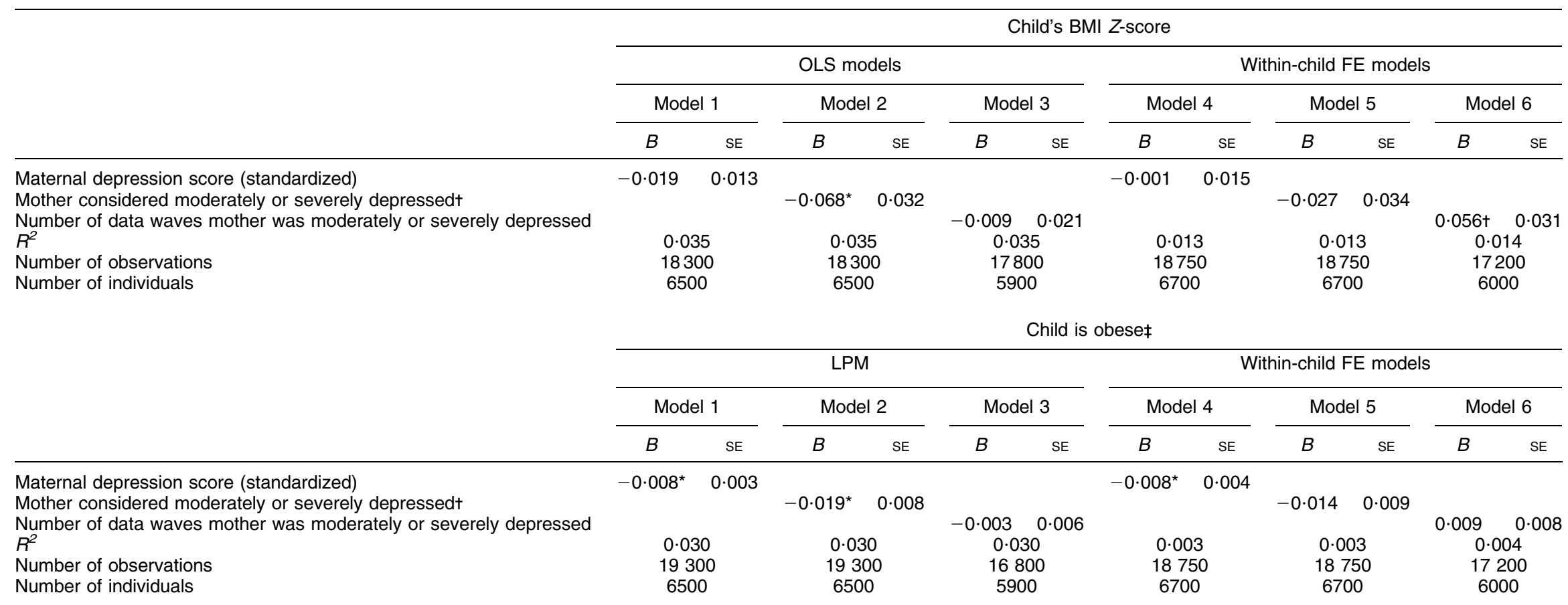

NCES, National Center for Education Statistics; CIDI-SF, Composite International Diagnostic Interview Short Form; CES-D, Center for Epidemiologic Studies Depression Scale; CDC, Centers for Disease Control and

Prevention.
Child and family covariates (not shown) are controlled: child age, gender, race, ethnicity, health insurance coverage, mothers' pre-pregnancy weight, maternal and paternal employment hours, family structure, parental education, family poverty status, and data collection wave. Sample sizes are rounded to the nearest fifty, in accordance with NCES regulations. Within $R^{2}$ are provided for the FE models.

At the 2-year wave, mothers reporting $\geq 1$ affirmative answer to the three stem questions on the CIDI-SF were considered moderately or severely depressed. At all other waves, mothers scoring $\geq 9$ on the CES-D were considered moderately or severely depressed.

using the percentiles for length-for-weight for measures taken before 24 months and weight-for-height (i.e. BMI) at 24 months and older, according to CDC guidelines (at or above the 95th ercentile for age and sex) 
Table 3 Predicting children's healthy and unhealthy food consumption from measures of maternal depression: ordinary least squares (OLS) and within-child fixed-effects (FE) models; Early Childhood Longitudinal Study-Birth Cohort, USA, 2001-2007

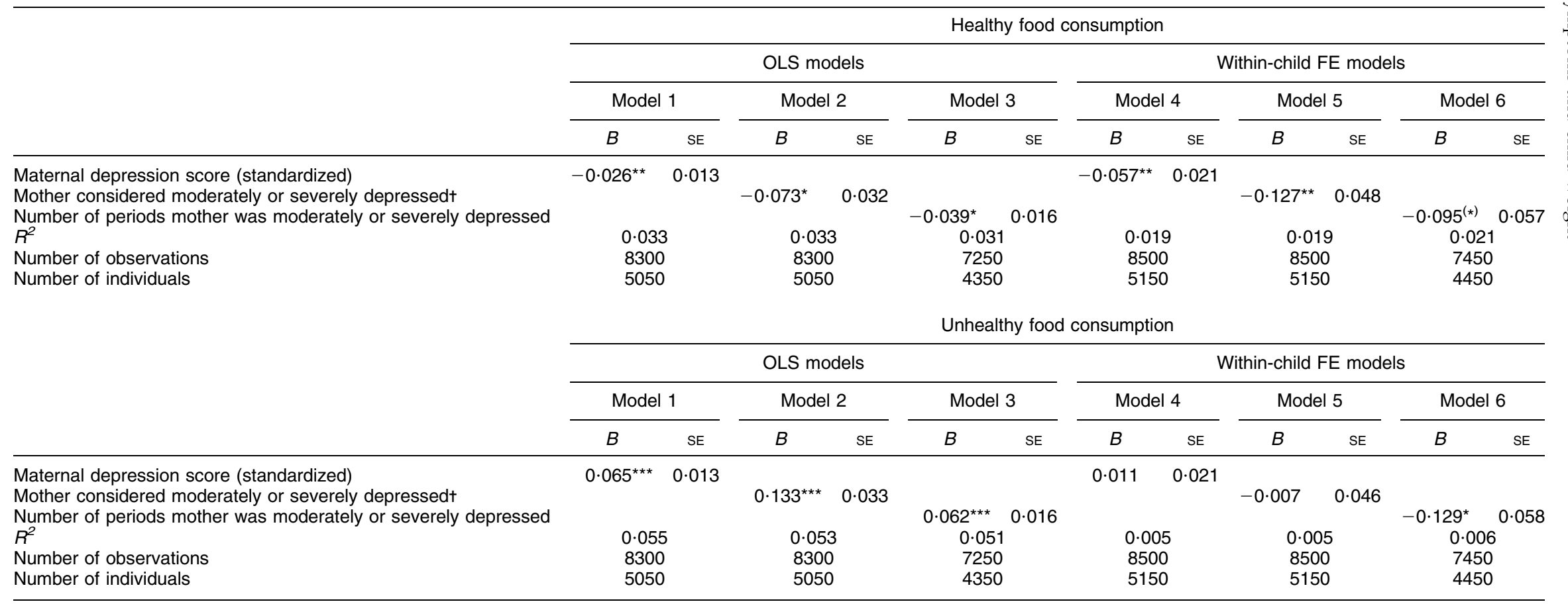

NCES, National Center for Education Statistics; CIDI-SF, Composite International Diagnostic Interview Short Form; CES-D, Center for Epidemiologic Studies Depression Scale.

Child and family covariates (not shown) are controlled: child age, gender, race, ethnicity, health insurance coverage, mothers' pre-pregnancy weight, maternal and paternal employment hours, family structure, parental education, family poverty status, and data collection wave. Sample sizes are rounded to the nearest fifty, in accordance with NCES regulations. Within $R^{2}$ are provided for the FE models.

tAt the 2 year wave, mothers reporting $\geq 1$ affirmative answer to the three stem questions on the CIDI-SF were considered moderately or severely depressed. At all other waves, mothers scoring $\geq 9$ on the CES-D were considered moderately or severely depressed. 
associations, suggesting there is no cumulative effect of exposure to moderate or severe maternal depressive symptoms on children's later weight and eating outcomes.

\section{Discussion}

The present study used longitudinal analyses to examine the associations of maternal depressive symptoms with children's standardized BMI, obesity and food consumption. In general, results suggest that maternal depressive symptoms are associated with a small decrease in the likelihood her child is obese and lower consumption of healthy foods among young children. While these associations were statistically significant, it is important to recognize their small magnitude. An increase of $1 \mathrm{SD}$ in maternal depressive symptoms scores, a substantial increase, was associated with less than a 1 percentage point decrease in the likelihood her child is obese, a result that arguably has little clinical significance. Further, it is important to note that, despite high childhood obesity rates and their negative consequences, maternal depressive symptoms should not be considered beneficial to children's weight, but rather the negative associations identified in the present study may indicate issues regarding healthy growth, eating or physical activity during early childhood.

Whereas measures of maternal depressive symptoms were associated with a lower likelihood of obesity in LPM models and greater unhealthy food consumption in OLS models, these associations were more limited in FE analyses, which arguably provide more robust estimates. Findings from the present study suggest that omitted variable bias may underlie some of the mixed findings identified in the existing literature ${ }^{(19-21,27-29)}$ and demonstrate the importance of controlling for confounding factors when investigating the effects of maternal depressive symptoms on child health and development. Furthermore, findings highlight the importance of controlling for measures of maternal mental health when examining children's health outcomes. Alternatively, the fewer associations in the FE models may result from these models themselves such that they associate changes in predictors with changes in outcomes. It is possible that instability in mothers' depressive symptoms does not lead to changes in children's weight outcomes, but rather that exposure to maternal depressive symptoms has a contemporaneous effect on children's weight. Furthermore, previous work has found that maternal sensitivity and depressive symptoms are associated with children's overweight during the school-age period ${ }^{(28)}$; thus, the effects of maternal depressive symptoms on children's weight may not appear until after kindergarten entry, the oldest age of our sample.

Indeed, the positive coefficients between the duration of moderate and severe maternal depressive symptoms and increased child obesity were approaching significance, and potentially indicate that exposure to persistent maternal depressive symptoms may lead to increased overweight or obesity in elementary-school children, as found in other studies ${ }^{(28,29)}$. Furthermore, the present study found that persistent exposure was important for the weight outcomes for the sub-sample of children in less-educated families. Specifically, the duration a child was exposed to moderate or severe maternal depressive symptoms was associated with a small increase in BMI Z-score (0.08 SD) among children whose parents lack a college degree, suggesting that children in families with fewer educational resources may be more vulnerable to poorer weight outcomes as a result of sustained maternal depressive symptoms. Given that in 2010 fewer than 20\% of Americans over 25 years had a bachelor's degree or higher $^{(39)}$, these findings have implications for the majority of the US population. Results highlight a need for research to identify the mechanisms underlying this association and for community and mental health services to identify and reach these families to promote healthy behaviours.

Consistent with previous work indicating that maternal depression negatively impacts parenting and feeding practices $^{(10,15,41)}$ and that unhealthy eating may partially underlie links between maternal depression and children's weight ${ }^{(32)}$, our study found associations between mothers' depressive symptoms and their children's lower consumption of healthy foods across analysis models. Notably, however, our measure of children's food consumption was predominantly mother-reported, did not assess serving size and had low reliability, which may account for its lack of predictive value for children's weight outcomes. Furthermore, we lacked information on parents' food consumption, which likely influences children's behaviour and outcomes. However, to the extent that parental behaviours are stable, they are controlled in the FE models.

As with all research, our study had several limitations. First, although we used rigorous statistical techniques to limit omitted variable bias, we cannot draw causal conclusions between maternal depressive symptoms and children's weight outcomes. Second, we used self-reported measures of depressive symptoms, as opposed to clinical diagnoses of maternal depression, which may be more highly related to children's weight outcomes. As described above, the data had imperfect measures of children's food consumption. Third, the study suffered from attrition and non-response, and due to our use of pooled data across waves, appropriate sampling weights were unavailable. Finally, we used the weight and height standards recommended by the Centers for Disease Control and Prevention; other studies may use the WHO standards, which are based on a broader sample of children ${ }^{(40)}$.

\section{Conclusion}

The present study is one of the first in the USA to longitudinally examine the associations between maternal 
depressive symptoms and young children's BMI, obesity and food consumption. Results suggest an association between maternal depressive symptoms and small decreases in the likelihood her child is obese and in her child's consumption of healthy foods. Findings reveal significant but small, positive associations between persistent maternal depressive symptoms and children's obesity among those families in which parents lack a college degree. Together with the previous literature, these findings highlight the importance of identifying and treating maternal depressive symptoms early in a child's life. Future research should examine the mechanisms underlying these differences in families by educational attainment, including meal routines, physical activity or stress-induced metabolic changes.

\section{Acknowledgements}

Sources of funding: This project was supported by grant number R40MC23630 from the Maternal and Child Health Bureau of the Health Services and Resources Administration, US Department of Health and Human Services. Its contents are solely the responsibility of the authors and do not necessarily represent the official views of the Department of Health and Human Services. The Department of Health and Human Services had no role in the design, analysis or writing of this article. Conflicts of interest: None. Ethical approval: The Institutional Review Boards of American University and the University of Maryland exempted this research. Authors contributions: T.W.M. led the conceptual design of the study, secured the project funding as the Principal Investigator, conducted the statistical analyses, drafted the majority of the initial manuscript, and approved the final manuscript as submitted. R.K.D. contributed to the project's conception, secured the project funding as the Co-Principal Investigator, wrote the discussion and conclusion sections, edited the manuscript, and approved the final manuscript as submitted. Acknowledgements: The authors would like to thank participants at colloquia at the 2012 American Public Health Association and the 2013 Society for Research in Child Development conferences for their comments on earlier versions of this research.

\section{Supplementary material}

To view supplementary material for this article, please visit http://dx.doi.org/10.1017/S1368980013003376

\section{References}

1. Ogden CL, Carroll MD, Kit BK et al. (2012) Prevalence of obesity and trends in body mass index among US children and adolescents, 1999-2010. JAMA 307, 483-490.

2. Kuczmarski RJ, Ogden CL, Guo SS et al. (2002) 2000 CDC growth charts for the United States: methods and development. Vital Health Stat 11, issue 246, 1-190.
3. Institute of Medicine (2005) Preventing Childhood Obesity: Health in the Balance. Washington, DC: National Academies Press.

4. Whitaker RC, Wright JA, Pepe MS et al. (1997) Predicting obesity in young adulthood from childhood and parental obesity. New Engl J Med 337, 869-873.

5. Wang Y \& Beydoun MA (2007) The obesity epidemic in the United States - gender, age, socioeconomic, racial/ ethnic, and geographic characteristics: a systematic review and meta-regression analysis. Epidemiol Rev 29, 6-28.

6. Institute of Medicine (2011) Early Childhood Obesity Prevention Policies. Washington, DC: National Academies Press.

7. Bianchi SM (2000) Maternal employment and time with children: dramatic change or surprising continuity? Demography 37, 401-414.

8. Committee on Integrating the Science of Early Childhood Development, Board on Children, Youth, and Families, National Research Council \& Institute of Medicine (2000) From Neurons to Neighborhoods: The Science of Early Childhood Programs [JP Shonkoff and DA Phillips, editors]. Washington, DC: National Academies Press.

9. Murray L, Fiori-Cowley A, Hooper R et al. (1996) The impact of postnatal depression and associated adversity on early mother-infant interactions and later infant outcome. Child Dev 67, 2512-2526.

10. McLearn KT, Minkovitz CS, Strobino DM et al. (2006) The timing of maternal depressive symptoms and mothers' parenting practices with young children: implications for pediatric practice. Pediatrics 118, e174-e182.

11. Sills MR, Shetterly S, Xu S et al. (2007) Association between parental depression and children's health care use. Pediatrics 119, e829-e836.

12. Vesga-Lopez O, Blanco C, Keyes K et al. (2008) Psychiatric disorders in pregnant and postpartum women in the United States. Arch Gen Psychiatry 65, 805-815.

13. Halbreich U \& Karkun S (2006) Cross-cultural and social diversity of prevalence of postpartum depression and depressive symptoms. J Affect Disord 91, 97-111.

14. McConley RL, Mrug S, Gilliland MJ et al. (2011) Mediators of maternal depression and family structure on child BMI: parenting quality and risk factors for child overweight. Obesity (Silver Spring) 19, 345-352.

15. McLearn KT, Minkovitz CS, Strobino DM et al. (2006) Maternal depressive symptoms at 2 to 4 months post partum and early parenting practices. Arch Pediatr Adolesc Med 160, 279-284.

16. American Academy of Pediatrics (2012) Breastfeeding and the use of human milk. Pediatrics 129, e827-e841.

17. Luppino FS, De Wit LM, Bouvy PF et al. (2010) Overweight, obesity, and depression: a systematic review and metaanalysis of longitudinal studies. Arch Gen Psychiatry 67, 220-229.

18. Siervo M, Wells JCK \& Cizza G (2009) The contribution of psychosocial stress to the obesity epidemic: an evolutionary approach. Horm Metab Res 41, 261-270.

19. Casey P, Goolsby S, Berkowitz C et al. (2004) Maternal depression, changing public assistance, food security, and child health status. Pediatrics 113, 298-304.

20. Surkan P, Kennedy C, Hurley K et al. (2011) Maternal depression and early childhood growth in developing countries: systematic review and meta-analysis. Bull World Health Organ 89, 607-615.

21. Rahman A, Iqbal Z, Bunn J et al. (2004) Impact of maternal depression on infant nutritional status and illness: a cohort study. Arch Gen Psychiatry 61, 946-952.

22. Anoop S (2004) Maternal depression and low maternal intelligence as risk factors for malnutrition in children: a community based case-control study from South India. Arch Dis Child 89, 325-329. 
23. Surkan PJ, Kawachi I, Ryan LM et al. (2008) Maternal depressive symptoms, parenting self-efficacy, and child growth. Am J Public Health 98, 125-132.

24. Surkan PJ, Kawachi I \& Peterson KE (2008) Childhood overweight and maternal depressive symptoms. I Epidemiol Community Health 62, e11.

25. Grote V, Vik T, Von Kries R et al. (2010) Maternal postnatal depression and child growth: a European cohort study. BMC Pediatrics 10, 14

26. Ajslev TA, Andersen CS, Ingstrup KG et al. (2010) Maternal postpartum distress and childhood overweight. PLoS One 5, e11136.

27. Ertel KA, Koenen KC, Rich-Edwards JW et al. (2010) Maternal depressive symptoms not associated with reduced height in young children in a US prospective cohort study. PloS One 5, e13656.

28. Wang L, Anderson JL, Dalton WT et al. (2013) Maternal depressive symptoms and the risk of overweight in their children. Matern Child Health J 17, 940-948.

29. Surkan PJ, Ettinger AK, Ahmed S et al. (2012) Impact of maternal depressive symptoms on growth of preschooland school-aged children. Pediatrics 130, e847-e855.

30. Grigsby-Toussaint DS, Chi S-H \& Fiese BH (2011) Where they live, how they play: neighborhood greenness and outdoor physical activity among preschoolers. Int J Health Geogr 10, 66.

31. Powell L \& Bao Y (2009) Food prices, access to food outlets and children's weight. Econ Hum Biol 7, 64-72.

32. Duarte CS, Shen S, Wu P et al. (2012) Maternal depression and child BMI: longitudinal findings from a US sample. Pediatr Obes 7, 124-133.
33. Fiese BH, Hammons A \& Grigsby-Toussaint D (2012) Family mealtimes: a contextual approach to understanding childhood obesity. Econ Hum Biol 10, 365-374.

34. Monsivais P, Mclain J \& Drewnowski A (2010) The rising disparity in the price of healthful foods: 2004-2008. Food Policy 35, 514-520.

35. Sturm R \& Datar A (2011) Regional price differences and food consumption frequency among elementary school children. Public Health 125, 136-141.

36. Rockett HR, Breitenbach M, Frazier AL et al. (1997) Validation of a youth/adolescent food frequency questionnaire. Prev Med 26, 808-816.

37. Radloff LS (1977) The CES-D: a self-report depression scale for research in the general population. Appl Psychol Meas 1, 385-401.

38. Kessler R, Barker PR, Colpe LJ et al. (2003) Screening for serious mental illness in the general population. Arch Gen Psychiatry 60, 184-189.

39. US Census Bureau (2012) Educational Status in the United States: 2010 - Detailed Tables. http://www.census.gov/ hhes/socdemo/education/data/cps/2010/tables.html (accessed December 2013).

40. De Onis M, Onyango AW, Borghi E et al. (2007) Comparison of the World Health Organization (WHO) child growth standards and the National Center for Health Statistics/WHO international growth reference: implications for child health programmes. Public Health Nutr 9, 942-947.

41. Morrissey TW (2013) Maternal depressive symptoms and weight-related parenting behaviors. Matern Child Health $J$ (In the Press). 